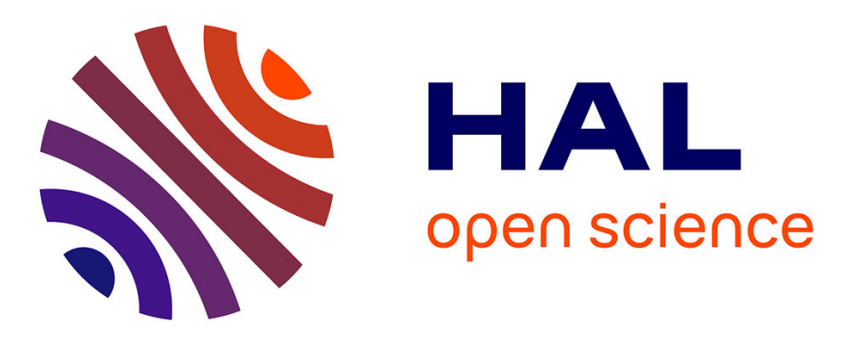

\title{
Development of a ion-exchanged glass integrated optics DFB laser for a LIDAR application
}

\author{
Lionel Bastard, Jean-Emmanuel Broquin, Florent Gardillou, Cedric
}

Cassagnettes, Jean-Pierre Schlotterbeck, Philippe Rondeau

\section{To cite this version:}

Lionel Bastard, Jean-Emmanuel Broquin, Florent Gardillou, Cedric Cassagnettes, Jean-Pierre Schlotterbeck, et al.. Development of a ion-exchanged glass integrated optics DFB laser for a LIDAR application. SPIE Photonics West, International Society for Optics and Photonics, Feb 2009, San Jose, CA, United States. pp.721817, 10.1117/12.811881 . hal-01963846

\section{HAL Id: hal-01963846 \\ https://hal.univ-grenoble-alpes.fr/hal-01963846}

Submitted on 11 Oct 2019

HAL is a multi-disciplinary open access archive for the deposit and dissemination of scientific research documents, whether they are published or not. The documents may come from teaching and research institutions in France or abroad, or from public or private research centers.
L'archive ouverte pluridisciplinaire HAL, est destinée au dépôt et à la diffusion de documents scientifiques de niveau recherche, publiés ou non, émanant des établissements d'enseignement et de recherche français ou étrangers, des laboratoires publics ou privés. 


\section{Development of a ion-exchanged glass integrated optics DFB laser for a LIDAR application}

Lionel Bastard, Jean-Emmanuel Broquin, Florent Gardillou, Cedric Cassagnettes, Jean-Pierre Schlotterbeck, et al.

Lionel Bastard, Jean-Emmanuel Broquin, Florent Gardillou, Cedric Cassagnettes, Jean-Pierre Schlotterbeck, Philippe Rondeau, "Development of a ion-exchanged glass integrated optics DFB laser for a LIDAR application," Proc. SPIE 7218, Integrated Optics: Devices, Materials, and Technologies XIII, 721817 (9 February 2009); doi: 10.1117/12.811881

Event: SPIE OPTO: Integrated Optoelectronic Devices, 2009, San Jose, California, United States 


\title{
Development of an ion-exchanged glass integrated optics DFB laser for a LIDAR application
}

\author{
Lionel Bastard*a, Jean-Emmanuel. Broquin ${ }^{\mathrm{a}}$, \\ Florent Gardillou ${ }^{\mathrm{b}}$, Cedric Cassagnettes ${ }^{\mathrm{b}}$, Jean-Pierre Schlotterbeck ${ }^{\mathrm{c}}$, Philippe Rondeau ${ }^{\mathrm{c}}$ \\ aIMEP-LaHC institute, 3 Parvis Louis Néel, 38000 Grenoble, France; \\ ${ }^{\mathrm{b}}$ Teem-Photonics S.A., 61 chemin du Vieux Chêne, 38240 Meylan, France ; \\ ${ }^{\mathrm{c}}$ Thalès avionics S.A., 25 rue Jules Védrines, 26000 Valence, France
}

\begin{abstract}
In this paper, we present the development of a laser source for a LIDAR application. This sensor is proposed as a standby instrument to provide a way to measure some aircraft air data such as the air speed. Although such systems already exist, none of them are based on an optical measurement. Thus, the use of a LIDAR would provide a backup channel with different failure modes than existing systems. Our LIDAR system allows determining the air speed through Doppler measurement at a wavelength of $1.55 \mu \mathrm{m}$ on aerosol particles present around the aircraft. The core of this device is a glass integrated optics continuous DFB laser. Its performances in term of single-frequency, stability, noise and linewidth are assessed in order to ensure the correct operation of the LIDAR system.
\end{abstract}

Keywords: glass integrated optics, aeronautics, LIDAR, DFB laser, erbium-ytterbium doped glass, distributed phase shift

\section{INTRODUCTION}

Optical sensors are nowadays used for a variety of applications ranging from medical diagnostic [1] to metrology [2]. In this study, we are interested in developing an optical sensor that can evaluate the true air speed (TAS) of an aircraft. Sensors realizing this function already exist: Pitot tubes, which are measuring differential pressure, are indeed placed at strategic points of the aircraft fuselage in order to measure TAS; the Global Positioning System (GPS) can also be used to determine the TAS. Since there are two ways to measure the aircraft TAS, there should be no need for an alternative measurement using an optical sensor. However, it must be noticed that the TAS is a crucial data that is mandatory to the flight safety, this is why the aircraft industry strongly encourages the development of a third TAS measurement channel, which would present different failure modes than the GPS and the Pitot tubes.

In this study, the use of a LIDAR system in order to measure the air speed is presented. LIDAR is an acronym that stands for "LIght Detection And Ranging" and is the extension of RADAR to lightwave frequencies. Usually, LIDAR systems are mostly used to detect particles in meteorology and atmospheric physics [3], to detect solid objects in oceanography, geology or even to realize landscapes mappings [4]. Two different practical implementations of LIDAR systems are generally found in the literature. The first one uses a pulsed light source in order to measure the time of flight between the sensor and the object [3]. This kind of sensor can thus be used for ranging or for measuring the velocity of slow objects. The other kind of LIDAR sensor is based on the Doppler effect. In this case, the light source can be either continuous or pulsed and the system detects the frequency shift of the back-reflected beam. This frequency shift can then be linked to the axial speed of the reflecting object by the well-known Doppler relation:

$$
\Delta v=-\frac{2 V}{\lambda}
$$

bastard@minatec.inpg.fr; phone +33 456529530; http://imep-lahc.grenoble-inp.fr/

Integrated Optics: Devices, Materials, and Technologies XIII, edited by Jean-Emmanuel Broquin, Christoph M. Greiner, Proc. of SPIE Vol. 7218, 721817 · @ 2009 SPIE · CCC code: 0277-786X/09/\$18 · doi: 10.1117/12.811881 
Where $\Delta v$ is the Doppler induced frequency shift of the back-reflected lightwave, $V$ is the axial speed of the object and $\lambda$ is the light source wavelength. In our case, if the aircraft speed is $100 \mathrm{~m} / \mathrm{s}$, the frequency shift of the reflected light would be around $30 \mathrm{MHz}$ if we use a $1.55 \mu \mathrm{m}$ wavelength incident light.

The Doppler detection can thus enable the measurement of air speed using the reflection of light by the particles present in the air around the aircraft. Several experiments were carried out to realize such a measurement: most of the airborne LIDAR systems were implemented on helicopters [5] where the altitude is lower so that the aerosol particle density is higher. However, some systems were implemented on aircrafts [6] using high power $\mathrm{CO}_{2}$ lasers. The wavelength of 10 $\mu \mathrm{m}$ emitted by $\mathrm{CO}_{2}$ laser is great compared to the aerosol particle size, entailing a low back-reflection efficiency, but the high amount of optical power available from these lasers did compensate for it.

Based on these successful experiments, a project called NESLIE was initiated by Thalès Avionics and funded by the European Union to develop a LIDAR prototype that could be implemented on civilian or military aircrafts as a backup air data channel. The main concern being safety, the chosen operating wavelength was the eye-safe region: $1.55 \mu \mathrm{m}$. The laser source was chosen to be a glass integrated optics laser source, which provides both a very low RIN in the MHz region and a narrow linewidth [7]. These characteristics are indeed of major importance for this application. Moreover, the choice of this type of source leads to a more compact device and has a better potential of integration when compared to optical fiber-based devices.

In the first section of this paper, we present the architecture of the LIDAR anemometer developed in the frame of the NESLIE project. Then, we focus on the conception and realization of the laser source that is the core of the LIDAR device. The last section is devoted to the characterization of the DFB laser on glass that has been developed.

\section{ARCHITECTURE OF THE LIDAR ANEMOMETER}

The proposed architecture for the LIDAR is depicted on figure 1. As an optical source we use a laser emitting at a wavelength of $1.55 \mu \mathrm{m}$. There are two main reasons for this choice: first, the availability of efficient high-power pump diodes, which were developed for optical communication amplification systems; the second and main reason to choose this wavelength for the laser operation is that it is located in the eye-safe region which solves hence a lot of laser safety issues when the aircraft is landed.

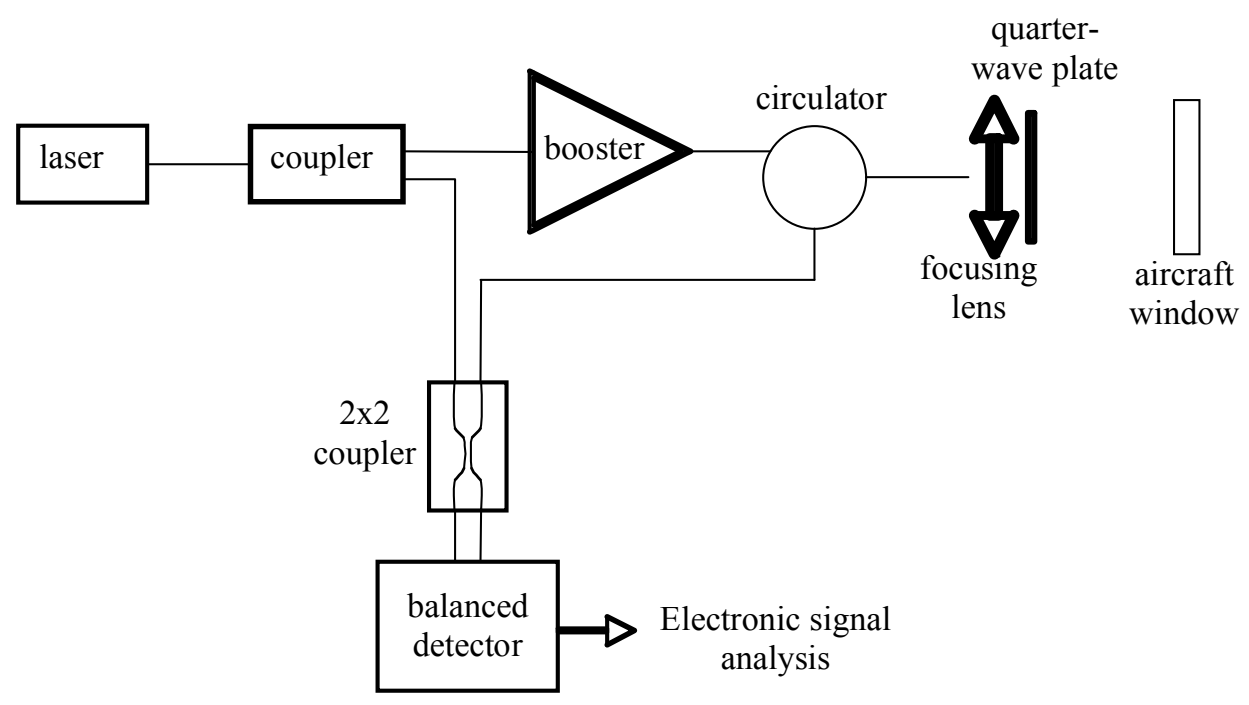

Figure 1.: LIDAR architecture

The output of the laser is first split in two using a directional coupler. The first half of the laser source power is amplified by a booster and then launched in free space via an optical circulator. The free space optical components are composed of a quarter-wave plate followed by a focusing lens. This assembly is used on one hand, to create a circularly polarized 
beam, which is focused outside of the aircraft; on the other hand, it is also used to collect the back-reflected light and to couple it back in the optical fiber as a linearly polarized light. Because of the quarter-wave plate, the polarization of the back-coupled beam is perpendicular to the one of the beam delivered by the laser. The circulator transfers the backcoupled light to a $2 \times 2$ coupler connected to a balanced detector. The second half of the laser output power is directly injected to the other input of the $2 \times 2$ coupler and acts as a local oscillator on the detector. The $2 \times 2$ coupler and the detector allow both optical signals (the local oscillator and the back-reflected signal) to be mixed and create a RF signal which frequency is equal to the frequency difference between the two optical signals. Since this frequency shift is induced by the Doppler effect, it is proportional to the relative speed of the reflecting particle toward the aircraft.

\subsection{Optical power budget and laser output power requirements}

The limiting factor concerning the optical power budget is the fraction of the optical power that is reflected by the particle and coupled back into the optical fiber. This quantity, which depends on the size of the reflecting particles and on the laser wavelength, can be evaluated thanks to the Mie-Rayleigh diffusion model. At $\lambda=1.55 \mu \mathrm{m}$, the backreflection from molecules constituting the air (mainly $\mathrm{O}_{2}$ and $\mathrm{N}_{2}$ ) is too small. Therefore, it is necessary to rely on aerosol particles to reflect the light. Previous experiments [8] have indeed shown that these aerosol particles are present in the atmosphere with a size distribution at flight altitudes that can be approximated to a log-normal law where particle radii ranging from 0.05 to $1 \mu \mathrm{m}$ for densities of the order of a few particles per cubic centimeter. From these measurements, the fraction of power back-reflected by such particles would be in the order of $-90 \mathrm{~dB}$. Although, a more accurate estimation should take into account the numerical aperture of the collecting optics, we will use this rough value for our power budget.

From this $-90 \mathrm{~dB}$ reflection coefficient and assuming that the sensitivity of the photodetector is of the order of $1 \mathrm{nW}$ (limited by the shot noise of the local oscillator), the power launched on the particle should be of about 1 Watt. This power will be delivered by the optical booster, which is seeded by the laser. Since about $5 \mathrm{~mW}$ is needed to seed the booster and about $3 \mathrm{~mW}$ is needed to saturate the detector with the local oscillator, the overall output power of the laser should be around $10 \mathrm{~mW}$.

\subsection{Detection}

The detection is achieved by launching both the local oscillator and the particle signal in a $2 \times 2$ coupler linked to a balanced detector. Since a $3 \mathrm{~dB}$-coupler entails a $\pi$ phase-shift between its two outputs. The optical electric field at the end of the coupler can be written as:

$$
\begin{aligned}
& E_{1}=\frac{E_{L O} e^{-2 \pi j v t}}{\sqrt{2}}+\frac{E_{S} e^{-2 \pi j(v+\Delta v) t}}{\sqrt{2}} e^{j \pi} \\
& E_{2}=\frac{E_{L O} e^{-2 \pi j v t}}{\sqrt{2}} e^{j \pi}+\frac{E_{S} e^{-2 \pi j(v+\Delta v) t}}{\sqrt{2}}
\end{aligned}
$$

Where $\mathrm{E}_{1}$ denotes the electric component of the electromagnetic field present at the first output arm of the $2 \times 2$ coupler, and $E_{2}$ at the second output. The detectors located at both ends of the couplers detect the time-averaged optical powers which are respectively proportional to:

$$
\begin{aligned}
& \frac{1}{2} E_{L O}{ }^{2}+\frac{1}{2} E_{S}{ }^{2}+E_{L O} E_{S} \sin (2 \pi \Delta v \cdot t) \\
& \frac{1}{2} E_{L O}{ }^{2}+\frac{1}{2} E_{S}{ }^{2}-E_{L O} E_{S} \sin (2 \pi \Delta v \cdot t)
\end{aligned}
$$

Since the balanced detector is designed to realize the difference between the two detected optical powers, this configuration allows obtaining the beating term. This signal can then be processed in order to evaluate the frequency shift $\Delta v$ and thus compute the aircraft air speed.

\subsection{Narcisse noise elimination}

With a booster output of $1 \mathrm{~W}$, even a small reflection of this signal (called narcisse signal) can be considered as a high power when compared to the optical power reflected by the particles. This power in itself should not be a problem since it does not interfere with the beating signal between the local oscillator at the frequency $v$ and the air signal at $v+\Delta v$. However, if we take into account the Relative Intensity Noise (RIN) of the laser, it is no more the case. The RIN of the 
laser is indeed located at frequencies around the beating frequency $\Delta v$. Since it is injected on the same coupler arm as the measurement signal, the RIN of the narcisse signal will beat with the local oscillator. Moreover, even though this power is very low, it can be of the same order of magnitude of the one reflected by the particles. The quarter-wave plate associated to the polarizing fiber is used to cancel this effect. Indeed, The quarter-wave plate indeed ensures that the signal beam is polarized perpendicularly to the input beam. This signal then travels without losses through the polarizing fiber, whereas the narcisse signal is killed by the polarizing fiber.

\section{DEVELOPMENT OF THE LASER SOURCE}

The architecture of the LIDAR sensor entails several constraints on the laser. First of all, the laser must emit a single frequency. Several kinds of devices allow such operation, but only the quarter wavelength phase shifted DFB laser allows a robust strict single frequency operation. The second requirement derived from the architecture of the sensor is that the RIN of the laser should be very low over the range of frequencies where the Doppler measurement takes places, i.e. from $10 \mathrm{MHz}$ to $500 \mathrm{MHz}$. Moreover, the laser linewidth should be smaller than a few $\mathrm{MHz}$ in order not to hinder the measurement accuracy. These constraints tend to discard semiconductor DFB lasers which RIN peaks are placed between a few hundreds of $\mathrm{MHz}$ and a few $\mathrm{GHz}$ and which laser linewidth is usually higher than $10 \mathrm{MHz}$. Finally, the environment of the sensor has also to be taken into account. Since the LIDAR sensor is designed to be placed inside of an aircraft, insensitivity to vibrations should be as high as possible. This favors integrated technologies rather than fiber optics technologies. Summing all these constraints, the glass integrated technology appears to be suitable for this application. Glass integrated lasers were indeed demonstrated to have a strictly single frequency behavior, a RIN lower than $-150 \mathrm{~dB} / \mathrm{Hz}$ at $10 \mathrm{MHz}$ and a linewidth of $3 \mathrm{kHz}$ [7]. Using these performances as a starting point, we modified these glass lasers in order to have their performances meeting the needs of this LIDAR application.

The laser cavity must allow selecting a single emission frequency. For this reason, we chose a quarter phase-shifted DFB cavity design. The waveguides were realized by silver sodium ion-exchange in a commercially available erbiumytterbium co-doped phosphate glass. The Bragg grating needed for the DFB cavity was realized using an holographic exposure of photoresist followed by a reactive ion etching process to etch the grating pattern on the glass surface. In order to obtain the quarter wave phase shift, we tailored the waveguide design by increasing the waveguide width on its center [9].

The crucial point to obtain good output power efficiency is the setting of the interaction between the waveguide and the Bragg grating. Two technological parameters can be adjusted to tune this interaction: the waveguide burying depth and the grating depth. Figure 2 presents the results of simulations that have been carried-out using the coupled mode theory. The guided modes were assumed to be $3 \times 5 \mu \mathrm{m}$ Gaussian fields and the grating is $1 \mathrm{~cm}$ long. With these parameters, the value of the reflectivity at Bragg wavelength $\mathrm{R}_{\mathrm{B}}$ has been computed as a function of the waveguide burying depth (figure $2 a)$ and of the grating etching depth (figure $2 b$ ) respectively.
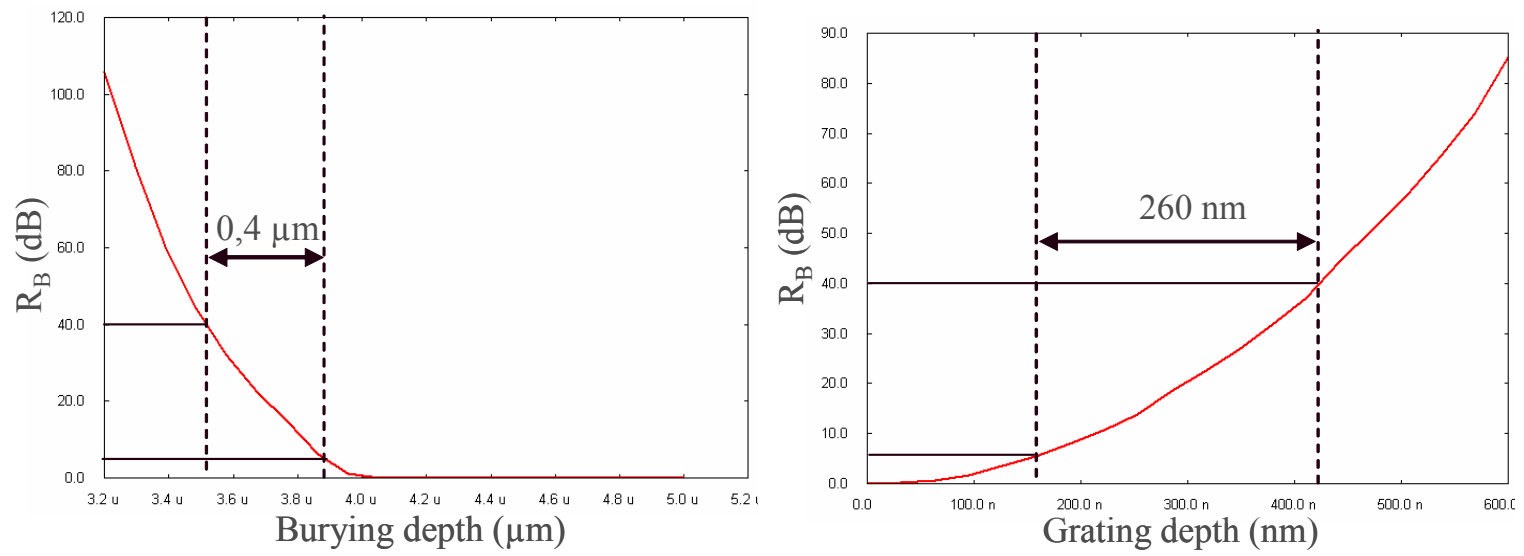

Figure 2: a) Variation of the grating reflectivity with the waveguide burying depth. b) Variation of the grating reflectivity with the grating etching depth 
As can be seen of figure 2a with a grating depth set to $\mathrm{xx} \mathrm{nm}$, the reflectivity evolves very quickly with burying depth. We can use as a guideline that the reflectivity should be kept between $5 \mathrm{~dB}$ and $40 \mathrm{~dB}$ in order to obtain a good laser feedback. Indeed, if the reflectivity is too low, the laser cavity will be too open entailing the use of a high amount of pump power to achieve lasing threshold; whereas, if the reflectivity is too high, propagation losses appear at the signal and pump wavelengths that prevent laser oscillation. Using theses guidelines, only a small span of burying depths ranging from 3.5 to $3.9 \mu \mathrm{m}$ can be used to produce a laser. Such small variations are difficult to control and almost impossible to measure directly.

For this reason, we chose to fix the burying time of the waveguides and to vary the burying current budget in order to obtain small but reproducible variations of the burying depth of the waveguides. Using this method, we produced several waveguides on which we realized identical Bragg gratings. We assessed the interaction efficiency between the waveguide and the grating by measuring the transmission spectra of the different samples. When a transmission spectrum with a suitable reflectivity was found, we kept this burying current budget and realized Bragg waveguides with different grating burying depths. This parameter indeed has a smaller influence over the waveguide-grating interactions and thus allowed us fine-tuning it. This time the optimization factor was the laser output power.

Finally, when we obtained lasing devices, only the phase shift design remained to be set. The photolithography mask we used for the waveguides possesses several phase shift designs. Using this, we have been able to select a phase shift design leading to a single frequency emission with the highest side mode suppression ratio. This design used a waveguide of $1.3 \mu \mathrm{m}$ with a phase shift section of $1.1 \mu \mathrm{m}$ width and $1 \mathrm{~mm}$ long. The grating depth was set to $150 \mathrm{~nm}$ and its length was $2 \mathrm{~cm}$.

\section{LASER CHARACTERIZATION}

One laser chip was realized using the optimal parameters presented in the previous section. The output facets were cut perpendicularly to the waveguides and polished with an angle of $8^{\circ}$ to ensure they cannot create additional feedback. We then performed a measurement of the fibre-coupled output power of the DFB laser versus launched pump power. The result of this measurement is displayed on figure 3. The output power was measured on the launched pump side of the device through a $980 \mathrm{~nm} / 1550 \mathrm{~nm}$ demultiplexer.
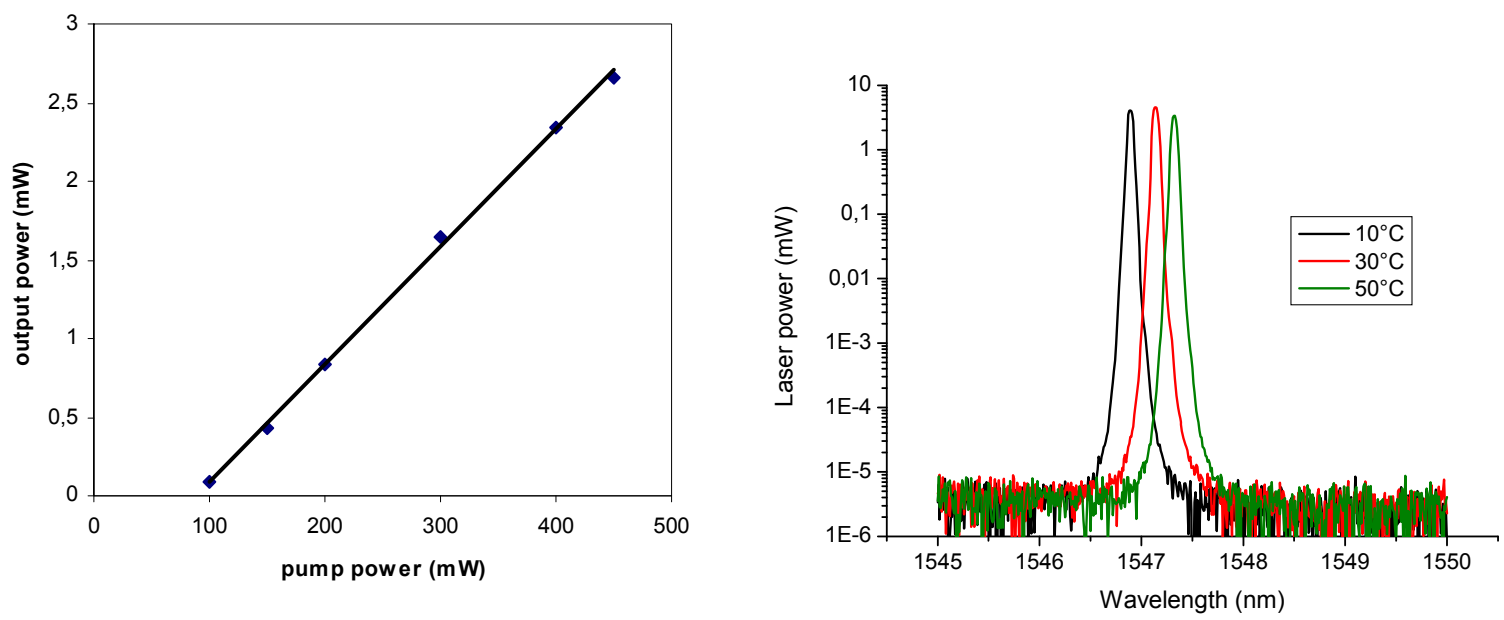

Figure 3: a) measurement of the output power versus pump power of the distributed phase shifted DFB laser. b) Measurement of the output spectrum using a $0.07 \mathrm{~nm}$ resolution OSA for three temperatures of operation.

The emission spectrum of the laser was checked using an optical spectrum analyser with a resolution $0.07 \mathrm{~nm}$ (see figure 3b). A single frequency peak was found which width could not be resolved by the measurement. However, the side mode suppression ratio (SMSR) can be determined from these measurements by calculating the difference between the laser 
peak power and the ASE ground level. Figure $3 \mathrm{~b}$ shows that the SMSR measurement was limited by the OSA sensitivity. For all lasers, a SMSR better than $60 \mathrm{~dB}$ were measured.

In order to ensure the strict single frequency operation of the laser, we performed a recirculating auto-heterodyne measurement of the laser signal using a RF spectrum analyser. This measurement allows measuring the linewidth of the laser [10]. The obtained result is displayed on figure 4. The $5 \mathrm{kHz}$ width of the Lorentzian Heterodyne beating signal correspond to a laser the linewidth of $2.5 \mathrm{kHz}$. Moreover, it can be noticed that the side lobes located at approximately $1.5 \mathrm{MHz}$ apart from the central peak correspond to the RIN relaxation peak.

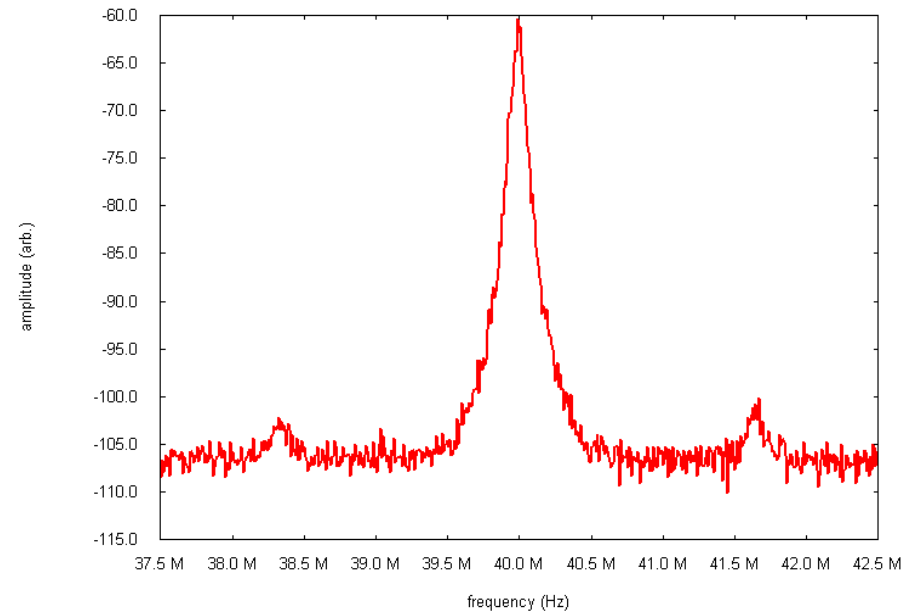

Figure 4: Measurement of the linewidth of the laser.

Finally, a measurement of the relative intensity noise of the laser has been carried-out. However, we did not measure the RIN in a standard configuration since for the LIDAR application, it is not the value of the RIN itself that is important, but the fact that it remains negligible when compared to the shot noise of the balanced detector that is actually limiting the Doppler signal measurement. The bench used for this characterization is depicted on figure 5 .

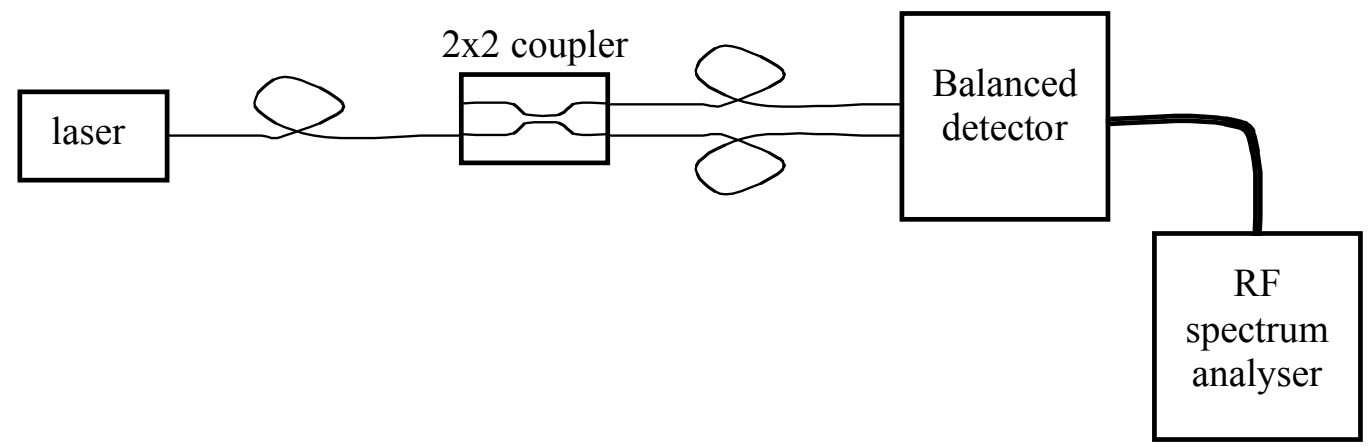

Figure 5: Bench used for the comparison of the laser RIN and the detection scheme shot noise.

To determine the ratio between the RIN of the laser and the shot noise of the balanced detector, we used a three measurements procedure. First, the measurement of the detector thermal noise $N_{t h}$. has been done with the laser turned off. For the second measurement, only one of the output ports of the $2 \times 2$ coupler has been connected to one of the input fibers of the balanced detector. The RF power $N_{\text {mono }}$ detected under this condition is theoretically given by:

$$
N_{\text {Mono. }}=N_{\text {th. }}+\frac{S N_{\text {Laser }}}{2}+\frac{\text { RIN }_{\text {Laser }}}{4}
$$


In this equation, $S N_{\text {Laser }}$ is the shot noise generated by the laser on the detector while $R I N_{\text {Laser }}$ is the relative intensity noise of the laser. Finally, the third measurement has been performed by connecting the second coupler output port to the second input port of the balanced detector. The RF spectrum analyser measurement gives:

$N_{\text {Bal.Det }}=N_{\text {th. }}+S N_{\text {Laser }}+C M R R \times R I N_{\text {Laser }}$

In this equation, CMRR denotes the common mode suppression ratio, which depends both on the optical power balancing of the two inputs of the balanced detector and on the electronics of the balanced detector. If the balancing is perfect, the CMRR term drops to zero. If the balancing is not good enough, one can artificially create some extra losses on one fiber port, for example by slightly bending one of the fibers. The ratio between the RIN and the shot noise can then be calculated by combining these three measurements, if the balanced measurement is realized with an optimum balancing so that the $C M R R$ term can be neglected:

$\frac{R I N_{\text {Laser }}}{S N_{\text {Laser }}}=\frac{4 N_{\text {Mono. }}-2 N_{\text {th. }}-2 N_{\text {Bal.Det. }}}{N_{\text {Bal.Det. }}-N_{\text {Det. }}}$

Figure 6 depicts the noise intensity measurements that has been obtained for the laser OFF, single canal and two canals balanced detection cases respectively.

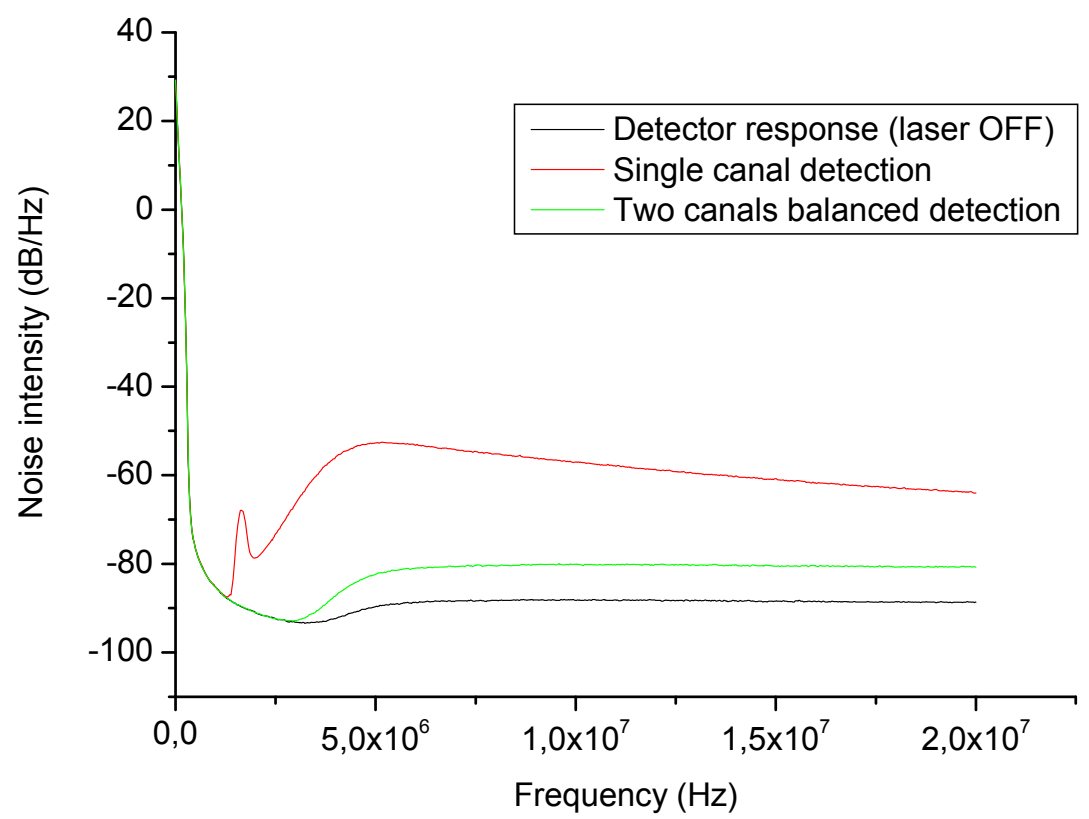

Figure 6: The three noise intensity measurements used to compute the ratio $\mathrm{RIN}_{\text {laser }} / \mathrm{SN}_{\text {laser }}$

The drop of all curves for frequencies below $5 \mathrm{MHz}$ is due to the high-pass filter of the detector electronics. Single canal detection shows a clear predominance of the RIN with its typical relaxation oscillation frequency peak close to $1.5 \mathrm{MHz}$. For the balanced detection measurement, the RIN peak vanishes and the noise intensity is drastically reduced. From these three measurements, the value of $\mathrm{RIN}_{\text {laser }} / \mathrm{SN}_{\text {laser }}$ was measured to be $34 \mathrm{~dB}$ at $5 \mathrm{MHz}$. This means that the CMRR of the balanced detector has to reach at least $44 \mathrm{~dB}$ in order to ensure the RIN of the laser remains $10 \mathrm{~dB}$ smaller then the detector noise and can be neglected. The specification given by the team in charge of assembling the whole sensor is a CMRR of $50 \mathrm{~dB}$. Thus, the value of $44 \mathrm{~dB}$ that we obtained shows that the RIN of the laser is low enough and even allows some margin for the LIDAR assembly.

\section{CONCLUSION}

In this paper, we presented a LIDAR sensor aimed at becoming a backup air data channel for civilian or military aircrafts. We first presented the overall architecture of the sensor which core is composed of a continuous wave DFB 
laser. Its output is split into two halves. One part is amplified and launched outside the aircraft to be reflected on aerosol particles. This signal is then launched together with the second half coming directly from the laser on a balanced detector. The Doppler frequency corresponding to the velocity of the particle can thus be measured. The laser was realized by silver-sodium ion exchange on a Erbium-Ytterbium phosphate glass. The laser cavity is realized by a Bragg grating etched on the glass surface, forming a DFB configuration. In order to ensure a single frequency operation, a quarter wave phase shift has been inserted at the center of the DFB laser cavity by modifying locally the waveguide width. The laser characterizations showed a fiber coupled output power of more than $10 \mathrm{dBm}$, a side mode suppression ratio better than $60 \mathrm{~dB}$ and a linewidth of $2.5 \mathrm{kHz}$. The effect of the intensity noise of the laser was also measured with the balanced detection configuration corresponding to the actual detection scheme of the sensor. It was shown that the RIN of the laser has a direct influence on the specification of the common mode suppression ratio of the balanced detector. In our case this CMRR has to be lower than $44 \mathrm{~dB}$, allowing a 6-dB margin when compared to the $50 \mathrm{~dB}$ specification.

\section{REFERENCES}

[1] Schult K, Katerkamp A, Trau D, Grawe F, Cammann K, Meusel M. "Disposable optical sensor chip for medical diagnostics: new ways in bioanalysis", Anal Chem., 71(23), 5430-5 (1999).

[2] Frank C, Gordon M.B., and Mumin S., "Overview of three-dimensional shape measurement using optical methods", Optical Engineering, 39(10) (2000)

[3] Shimizu H, Lee SA, She CY., "High spectral resolution lidar system with atomic blocking filters for measuring atmospheric parameters", Applied Optics, 22(9), 1373 (1983).

[4] Hudak A.T., Lefsky M.A., Cohen W.B., Berterretche M, "Integration of lidar and Landsat ETM+ data for estimating and mapping forest canopy height”, Remote Sensing of Environment, 82(2), 397-416 (2002)

[5] Bilbro, J., Fichtl G., Fitzjarrald D., Krause M., and Lee R., “Airborne Doppler Lidar Wind Field Measurements", Bull. Amer. Meteor. Soc., 65, 348-359 (1984)

[6] Köpp, F., Rahm S., and Smalikho I., "Characterization of Aircraft Wake Vortices by 2- $\mu$ m Pulsed Doppler Lidar", Journal of Atmospheric and Oceanic Technology, 21, 194-206 (2004)

[7] L. Bastard, S. Blaize, and J.-E. Broquin, "Glass integrated optics ultra narrow linewidth DFB lasers for DWDM applications," Optical Engineering 42(10), pp. 2800-2804, (2003)

[8] Wang P., Kent G., McCormick M., Thomason L., and Yue G., "Retrieval analysis of aerosol-size distribution with simulated extinction measurements at SAGE III wavelengths," Applied Optics 35, 433-440 (1996)

[9] Soda H., Wakao K., Sudo H., Tanahashi T., Imai H., "GaInAsP/InP phase-adjusted distributed feedback lasers with a step-like nonuniform stripe width structure," Electronics Letters, 20(24), 1016-1018 (1984)

[10] Lennartz C., Etten W.C., Osch T., and Huijskens F., "Laser Spectra Measured with the Recirculating Self Heterodyne Technique", Journal of Optical Communications, 17(4), 138-146 (1996) 